\title{
Erratum to: Phase II Trial of S-1 and Oxaliplatin Plus Cetuximab for Colorectal Cancer Patients with Initially Unresectable or Not Optimally Resectable Liver Metastases (KSCC1002)
}

Eiji Oki, MD, PhD, FACS ${ }^{1}$, Yasunori Emi, MD, $\mathrm{PhD}^{2}$, Yuji Miyamoto, MD, PhD ${ }^{3}$, Akira Kabashima, MD, PhD $^{4}$, Hidefumi Higashi, $\mathrm{MD}, \mathrm{PhD}^{5}$, Yutaka Ogata, $\mathrm{MD}, \mathrm{PhD}^{6}$, Masahiko Ikebe, MD, $\mathrm{PhD}^{7}$, Hiroshi Saeki, MD, PhD, FACS $^{1}$, Shoji Tokunaga, PhD $^{8}$, Ken Shirabe, MD, PhD, FACS ${ }^{1}$, Toru Beppu, MD, PhD ${ }^{3}$, Shinji Uchida, MD, PhD ${ }^{6}$, Mitsuhisa Takatsuki, MD, PhD ${ }^{9}$, Masahiko Sakoda, MD, PhD $^{10}$, Susumu Eguchi, MD, PhD, FACS ${ }^{9}$, Yoshito Akagi, MD, PhD ${ }^{11}$, Yoshihiro Kakeji, MD, PhD, FACS ${ }^{12}$, Hideo Baba, MD, PhD, FACS ${ }^{3}$, Shoji Natsugoe, MD, PhD ${ }^{10}$, Yoshihiko Maehara, MD, PhD, FACS ${ }^{1}$ and Kyushu Study Group of Clinical Cancer (KSCC)

${ }^{1}$ Department of Surgery and Science, Graduate School of Medical Sciences, Kyushu University, Fukuoka, Fukuoka, Japan; ${ }^{2}$ Department of Surgery, Saiseikai Fukuoka General Hospital, Fukuoka, Japan; ${ }^{3}$ Department of Gastroenterological Surgery, Graduate School of Medical Sciences, Kumamoto University, Kumamoto, Japan; ${ }^{4}$ Department of Surgery, Kyushu Central Hospital of the Mutual Aid Association of Public School Teachers, Fukuoka, Japan; ${ }^{5}$ Department of Surgery, Steel Memorial Yawata Hospital, Kitakyushu, Japan; ${ }^{6}$ Department of Surgery, Kurume University Medical Center, Kurume, Japan; ${ }^{7}$ Department of Gastroenterological Surgery, Beppu Medical Center, Beppu, Japan; ${ }^{8}$ Medical Information Center, Kyushu University Hospital, Fukuoka, Japan; ${ }^{9}$ Department of Surgery, Nagasaki University Graduate School of Biomedical Sciences, Nagasaki, Japan; ${ }^{10}$ Department of Digestive Surgery, Breast and Thyroid Surgery, Kagoshima University Graduate School of Medical and Dental Sciences, Kagoshima, Japan; ${ }^{11}$ Department of Surgery, Kurume University School of Medicine, Kurume, Japan; ${ }^{12}$ Division of Gastrointestinal Surgery, Department of Surgery, Graduate School of Medicine, Kobe University, Kobe, Japan

\section{ERRATUM TO: ANN SURG ONCOL DOI 10.1245/S10434-015-4771-1}

In first sentence of the Protocol Treatment section on the second page of the original article there is an error in the oxaliplatin dosage. The corrected sentence is as follows: In principle, 4-6 cycles of preoperative chemotherapy-SOX: $\mathrm{S}-1$ is orally administrated at a dose corresponding to the body surface area (BSA) $\left(40 \mathrm{mg}\right.$ with BSA $<1.25 \mathrm{~m}^{2}$; $50 \mathrm{mg}$ with BSA $1.25-1.50 \mathrm{~m}^{2} ; 60 \mathrm{mg}$ with BSA > $1.50 \mathrm{~m}^{2}$ ) twice daily after meals for 14 consecutive days, oxaliplatin $\left(130 \mathrm{mg} / \mathrm{m}^{2}\right)$ day 1 every 3 weeks, plus cetuximab $\left(400 \mathrm{mg} / \mathrm{m}^{2}\right.$ as the initial dose) followed by $250 \mathrm{mg} /$ $\mathrm{m}^{2} /$ week - was administered within 2 weeks after enrollment.

The online version of the original article can be found under doi:10.1245/s10434-015-4771-1.

(C) Society of Surgical Oncology 2015

Published Online: 21 Sep 2015

E. Oki, MD, PhD, FACS

e-mail: okieiji@surg2.med.kyushu-u.ac.jp 